\title{
Non-adiabatic asteroseismology of near-main sequence variable stars
}

\author{
M.-A. Dupret \\ Instituto de Astrofísica de Andalucía-CSIC, Apartado 3004, 18080 \\ Granada, Spain
}

\begin{abstract}
We present in this talk a new linear non-adiabatic pulsation code: $\mathrm{MAD}^{1}$. We detail all the possible applications of it in the frame of asteroseismology. By using it, photospheric observables such as multi-color photometric amplitude ratios, phase differences and line profile variations can be determined accurately and confronted with observations. Moreover, MAD enables the precisely analysis of the excitation mechanisms of pulsating stars. Applications to different kinds of variable stars ( $\delta$ Sct, $\beta$ Cephei, Slowly Pulsating B and $\gamma$ Dor stars) are presented by Dupret et al. (these proceedings), Handler \& Aerts (these proceedings), De Cat et al. (these proceedings) and De Ridder et al. (these proceedings).
\end{abstract}

\section{Non-adiabatic asteroseismology}

In the superficial layers of a star, the thermal relaxation time is shorter than the pulsation periods, so that the oscillations are highly non-adiabatic. The use of a non-adiabatic code is thus intrinsically necessary for an accurate confrontation with observables directly linked to the photosphere. The main specificity of MAD is the special care given to the treatment of the pulsation in the entire nongrey atmosphere. Hence, it is able to determine accurately the amplitudes and phases of variation of the different physical quantities in the very outer layers of stars. In particular, the normalized amplitude and phase of effective temperature variation can be determined. Its effect on line profile variations (Dupret et al. 2002; De Ridder et al. 2002) and on multi-color photometric amplitude ratios and phase differences (Dupret et al. 2003a) can be determined, which improves significantly the accuracy of mode identification methods. Moreover, since the non-adiabatic theoretical predictions are very sensitive to the characteristics of the equilibrium models, these models can be constrained by searching for the best fit between theory and observations; we term this method non-adiabatic asteroseismology.

\footnotetext{
${ }^{1}$ Our non-adiabatic code is available upon request from the author, email: madupret@iaa.es
} 


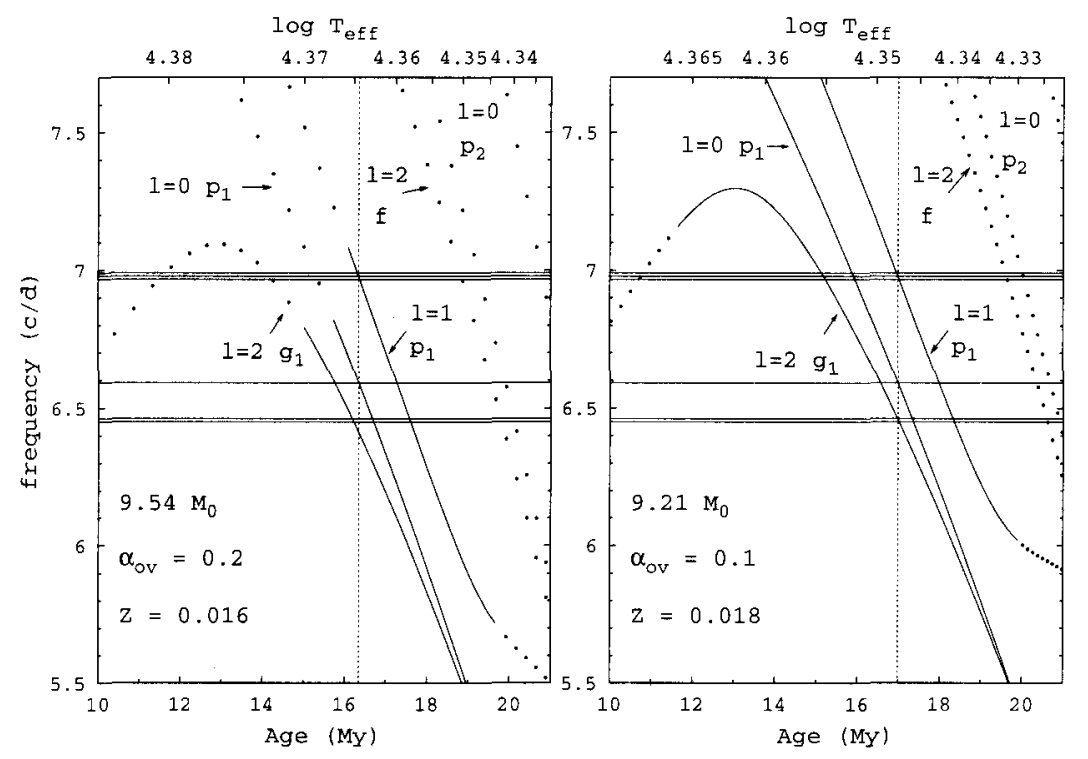

Figure 1. Evolution of the theoretical frequencies of HD 129929 models as a function of age and $\log T_{\text {eff }}$ for two different evolutionary sequences. The lines are the unstable modes and the dots are the stable ones.

\section{2. $\beta$ Cephei stars}

We first show the application of our non-adiabatic code to the $\beta$ Cephei star HD 129929. A huge observational effort has been performed for this star by different members of the Institute of Astronomy of Leuven. They gathered 1500 good-quality points over a total time base of $21.2 \mathrm{yr}$. From these observations they could detect at least 6 pulsation frequencies (Aerts et al. 2003a,b; Handler \& Aerts, these proceedings).

By confronting the observed Geneva photometric amplitude ratios to the predictions of our non-adiabatic code, we can identify without any doubt the degree $\ell$ of these modes as a radial mode, an $\ell=1$ triplet and two members of an $\ell=2$ quintuplet. Searching for models fitting exactly the observed frequencies allows us to relate the mass and the overshooting to the metallicity of the star. The last degree of freedom is then lifted by the non-adiabatic analysis. As shown, for example, in Dupret et al. (2003a), the theoretical non-adiabatic predictions (growth rates, photometric amplitude ratios and phase differences) are essentially sensitive to the metallicity of the models for $\beta$ Cep stars. On one hand, imposing that all the observed modes are excited, we find $Z \geq 0.016$. On the other hand, by confronting the observed photometric amplitude ratios with the theoretical predictions of our non-adiabatic code, we find a better agreement for models with $Z \leq 0.02$ than for the models with $Z>0.02$. We remark that these non-adiabatic predictions are in perfect agreement with the spectroscopic abundance determinations which give $Z=0.018 \pm 0.004$. 
By combining the fitting of the frequencies and the non-adiabatic analysis, we obtain the following constraints on the parameters of HD 129929, mass: $M \in$ $[9.0,9.5] \mathrm{M}_{\odot}$, overshooting: $\alpha_{\mathrm{ov}} \in[0.05,0.15]$, age $\in[16,18]$ Myr. In Fig. 1, we show the evolution of the theoretical frequencies (in $\mathrm{d} \mathrm{s}^{-1}$ ) of HD 129929 models as a function of age and $\log T_{\text {eff }}$ for two evolutionary sequences with different metallicities and overshootings. The lines are the unstable modes and the dots are the stable ones as determined by our non-adiabatic code. The horizontal lines are the observed frequencies. We see that the vertical line in the right panel corresponds to a model fitting exactly all the frequencies, and all of them are predicted to be unstable. We refer to Dupret et al. (2003b) for more details about the seismic modelling of HD 129929.

\section{Slowly Pulsating B stars}

The Slowly Pulsating B stars (gravity modes pulsators) can also be studied with our non-adiabatic code. We can analyse their driving mechanism, associated with the Fe partial ionization zone. MAD can also be used for the photometric mode identification of their dominant modes. We refer to De Cat et al. (these proceedings) for more details.

\section{4. $\delta$ Scuti stars}

The $\delta$ Scuti stars are also very good targets for asteroseismology. Difficulties in the modelling of these stars come from their often fast rotation and from their convective zones; we study here their convective zones. An important point is that the non-adiabatic predictions are very sensitive to the modelling of the thin convective envelope and to the treatment of its interaction with pulsation.

This sensitivity appears in the theoretically predicted photometric amplitude ratios and phase differences. Firstly, these observables are very sensitive to the value adopted for the mixing-length parameter $\alpha=l / H_{p}$ (Moya et al. these proceedings) and to the models of convection (MLT versus FST, Dupret et al. these proceedings). Secondly, the monochromatic flux and limb-darkening coefficients, which play a significant role in the determination of the photometric amplitudes and phases, are also very sensitive to the treatment of convection in the atmosphere models (Barban et al. 2003). And thirdly, the treatment of the time-dependent interaction between convection and pulsation has also a significant impact on the theoretical results (Dupret et al. in preparation). Therefore, by confronting the theoretical and observed photometric amplitudes and phases, constraints can be derived on the convective envelopes of $\delta$ Scuti stars.

We can also study the excitation mechanisms of $\delta$ Scuti stars. With the frozen convection version of our non-adiabatic code, we succeed in reproducing the blue edge of the $\delta$ Scuti instability strip; and with the time-dependent convection version of our code, we succeed in reproducing both the blue edge and the red edge of the instability strip (Dupret et al., these proceedings). 


\section{5. $\gamma$ Doradus stars}

The $\gamma$ Doradus stars are a recently-discovered class of variable stars with periods between $8 \mathrm{hr}$ and $3 \mathrm{~d}$. Guzik et al. (2000) proposed a driving mechanism for their gravity modes based on the blocking of the flux at the base of the convective envelope. However, in a significant part of the convective envelope, the frozen convection approximation adopted by Guzik et al. is not valid. With the last version of our non-adiabatic code, where we included time-dependent convection models, we succeed in explaining the driving of the $\gamma$ Dor g-modes in a consistent way. Our treatment agrees with the one of Guzik et al., in the sense that we also predict a blocking of the flux at the base of the convective envelope. But we no longer find driving in the partial ionization zones, because of the control by the convective flux. As a consequence, the range of unstable modes is slightly smaller for our time-dependent convection models than for frozen convection models.

\section{Conclusions}

We have presented the new non-adiabatic pulsation code MAD and applied it to different kinds of pulsating stars. This code can be used as a tool for mode identification and allows the constraint of stellar models of pulsating stars. For $\beta$ Cep stars, we showed that this code enables the derivation of constraints on the metallicity (independently of any spectroscopic analysis). This, together with the frequency fitting, allowed us to constrain the core overshooting of HD 129929. For $\delta$ Sct stars, constraints can be obtained on the models of the thin convective envelope. The driving of the $\gamma$ Dor stars can be explained by the theoretical predictions of our code. And finally, Slowly Pulsating B stars can also be studied.

Acknowledgments. This work was supported through a European Community Marie Curie Fellowship.

\section{References}

Aerts, C., Thoul, A., Daszyńska-Daszkiewicz, J., Scuflaire, R., Waelkens, C. et al. 2003a, Science, 300, 1926

Aerts, C. , Waelkens, C., Daszyńska-Daszkiewicz, J., Dupret, M.-A. et al. 2003b, $A \& A$, in press

Barban, C., Goupil, M. J., Van't Veer-Menneret, C., Garrido, R., Kupka, F., Heiter, U. 2003, A\&A, 405, 1095

De Ridder, J., Dupret, M.-A., Neuforge, C., Aerts, C. 2002, A\&A, 385, 572

Dupret, M.-A., De Ridder, J., Neuforge, C., Aerts, C., Scuflaire, R. 2002, A\&A, 385,563

Dupret, M.-A., De Ridder, J., De Cat, P., Aerts, C. et al. 2003a, A\&A, 398, 677

Dupret, M.-A., Thoul, A., Scuflaire, R., Aerts, C., Daszyńska-Daszkiewicz, J. et al. $2003 \mathrm{~b}, \mathrm{~A} \& \mathrm{~A}$, in press

Guzik, J. A., Kaye, A. B., Bradley, P. A., Cox, A. N., Neuforge, C. 2000, ApJ, $542, \mathrm{~L} 57$ 